\title{
Altered function of monocytes/macrophages in patients with autoimmune hepatitis
}

\author{
RUI LIN, JIE ZHANG, LU ZHOU and BANGMAO WANG \\ Department of Digestive Diseases, General Hospital, Tianjin Medical University, Tianjin 300052, P.R. China
}

Received April 1, 2015; Accepted February 4, 2016

DOI: $10.3892 / \mathrm{mmr} .2016 .4998$

\begin{abstract}
The pathogenesis of autoimmune hepatitis (AIH) involves the intervention of the innate and adaptive immune responses. In the current study, the alterations in monocytes/Kupffer cells (KCs) were investigated in patients with AIH. A total of 21 patients with $\mathrm{AIH}$ at different stages of the disease, and 7 controls with non-alcoholic fatty liver disease were selected. The abundance of VAV1 and p21-activated kinase 1 (PAK1) in the liver and KCs was analyzed. In addition, the expression levels of HLA-DR and CD80 in the peripheral blood monocytes (PBMs) were measured, and phagocytosis of PBMs was assessed. KCs of AIH patients exhibited higher expression levels of VAV1 and PAK1. This upregulated expression was associated with disease progression. A reduced expression of HLA-DR and CD80, and reduced capacity of E. coli phagocytosis in PBMs was observed for patients with AIH. This downregulated expression was associated with disease progression. The results of the current study indicated that defective function of KCs and PBMs may be involved in the pathogenesis of AIH.
\end{abstract}

\section{Introduction}

Autoimmune hepatitis (AIH) is a chronic inflammatory liver disorder with an etiology that remains unclear. The pathogenesis may be a result of alterations in immune tolerance, a genetic predisposition and environmental conditions, which in collaboration induce a T-cell-mediated attack on liver antigens, leading to necro-inflammation and liver damage (1). Monocytes/macrophages are a class of specialized antigen-presenting cells that

Correspondence to: Professor Bangmao Wang, Department of Digestive Diseases, General Hospital, Tianjin Medical University, 154 Anshan Road, Tianjin 300052, P.R. China

E-mail: wbangmao@126.com

Abbreviations: AIH, autoimmune hepatitis; KCs, Kupffer cells; NAFLD, non-alcoholic fatty liver disease; PAK1, p21-activated kinase 1; PBMs, peripheral blood monocytes

Key words: autoimmune hepatitis, Kupffer cells, peripheral blood monocytes, VAV1 serve an important role in the recruitment and activation of innate immune cells. Monocytes/macrophages are also able to deliver co-stimulatory signals to activate naive $\mathrm{T}$ cells, thus triggering the initiation of the adaptive immune responses. Therefore, they act as the bridge between the innate and adaptive immune systems. Previous studies indicate that dysfunction of monocytes/macrophages is important in the pathogenesis of numerous autoimmune diseases $(2,3)$, however the role of monocytes/macrophages in AIH remains unclear. Kupffer cells (KCs) are present throughout the liver, representing 80 90\% of all tissue macrophages in the body (4). Liver damage has been previously reported to result from the dysfunction of KCs (5).

Monocytes/macrophages serve three main functions including phagocytosis, antigen presentation and inflammatory cytokine production (6). Phagocytosis of pathogens or antigens is a central process in the host defense mechanism against infections and the immune responses (7). Antigen presentation is critical for activation of the adaptive immune response, and the process is closely associated with HLA-DR and CD80 expression levels $(8,9)$. The members of the ras homolog gene family (Rho) guanosine triphosphatase (GTPase) family are known to regulate signaling pathways leading to remodeling of the actin cytoskeleton, transcriptional regulation and the cell cycle. It has been suggested that the Rho GTPase family serves a critical role in cell adhesion, antigen presentation, migration, chemotaxis and phagocytosis (10). VAV1 and p21-activated kinase 1 (PAK1) have been previously described as effectors of the Rho GTPases $(11,12)$.

The aim of the current study was to measure the abundance of VAV1 and PAK1 in the livers of patients with AIH and further evaluate their expression in KCs. The expression levels of HLA-DR and CD80 in the peripheral blood monocytes (PBMs) of patients with AIH were also measured to assess antigen-presentation. In addition, the phagocytic functions of PBMs were evaluated by co-culture with fluorescent-labeled bacteria.

\section{Subjects and methods}

Patients. Subsequent to obtaining informed consent, 21 patients at the Department of Digestive Diseases, Tianjin Medical University General Hospital (Tianjin, China) with histologically confirmed AIH of different disease stages, were enrolled in the present study between January 2011 and February 2013. 
A total of 7 patients with non-alcoholic fatty liver disease (NAFLD), who were age- and gender-matched, were selected as the controls. The diagnosis of AIH was performed according to a simplified criteria for the diagnosis of AIH (13), and the diagnosis of NAFLD was performed according to the NAFLD guidelines by the American Gastroenterological Association, American Association for the Study of Liver Diseases and American Collage of Gastroenterology (14). The AIH patients included 2 men and 19 women with a mean age of $51.2 \pm 21.4$ years. The serum levels of alanine aminotransferase (ALT) were assessed using a commercially available kit (cat. no. 70911; normal level, $\leq 40$ U/1; Biobase, Shandong, China), and based on these results, and ultrasound or computerized tomography of the abdomen, the patients were classified into the AIH normal liver function group (ALT $\leq 40 \mathrm{U} / 1$, no cirrhosis), AIH abnormal liver function group (ALT $>40 \mathrm{U} / 1$, no cirrhosis) or the AIH cirrhosis group (cirrhosis). The 7 patients with NAFLD analyzed as the controls comprised 1 men and 6 women with a mean age of $53.1 \pm 19.9$ years. None of the patients had previously received any treatment.

The institutional review board and ethical committee of Tianjin Medical University General Hospital approved the study protocol. All patients provided written informed consent for their participation in the current study.

Western blotting of VAVI and PAK1. Liver tissue samples were obtained by ultrasound-guided percutaneous liver biopsy and stored at $-80^{\circ} \mathrm{C}$. Liver tissue samples were homogenized on ice in lysis buffer (Well-Biology, Changsha, China) containing $50 \mathrm{mM}$ Tris (pH 8.0), $150 \mathrm{mM} \mathrm{NaCl}, 1 \%$ Triton X-100, $100 \mu \mathrm{g} / \mathrm{ml}$ phenylmethanesulfonyl fluoride (Roche Diagnositcs, Basel, Switzerland). Lysates were clarified by centrifugation at $12,000 \mathrm{x}$ g and $4^{\circ} \mathrm{C}$ for $15 \mathrm{~min}$, and the protein concentration was determined using the Bicinchoninic Acid Assay kit (Thermo Fisher Scientific, Inc., Waltham, MA, USA). Supernatants were separated by $10 \%$ sodium dodecyl sulfate-polyacrylimide gel electrophoresis, and transferred onto a polyvinylidene difluoride membrane (EMD Millipore, Billerica, MA, USA). The membranes were blocked with $5 \%$ skimmed milk in phosphate-buffered saline (PBS; Well-Biology) for $1 \mathrm{~h}$, followed by incubation with the following primary antibodies overnight at $4^{\circ} \mathrm{C}$ : Mouse anti-glyceraldehyde 3-phosphate dehydrogenase (GAPDH) monoclonal antibody (1:1,000; sc-365062; Santa Cruz Biotechnology, Inc., Danvers, TX, USA); mouse anti-VAV1 polyclonal antibody (1:1,000; ab58106; Abcam, Cambridge, MA, USA); and rabbit anti-PAK1 monoclonal antibody (1:1,000; ab40852; Abcam). Subsequently, the membranes were incubated with horseradish peroxidase-conjugated goat anti-mouse (1:1,000; ZB-5305; ZSGB-BIO, Beijing, China) and goat anti-rabbit $(1: 1,000$; ZB-5301; ZSGB-BIO) IgG for $1 \mathrm{~h}$ at room temperature. The blots were detected using an enhanced chemiluminescence system (Syngene, Frederick, MD, USA).

Double immunostaining of CD68/VAV1 or PAK1. To characterize the expression of VAV1 and PAK1 on KCs, double-immunostaining for CD68 and either VAV1 or PAK1 was performed in the livers of patients with NAFLD and AIH. Sections were incubated with the monoclonal mouse anti-CD68 antibody (1:50; ab955; Abcam) and either polyclonal mouse anti-VAV1 (1:50) or monoclonal rabbit anti-PAK1 (1:50). Subsequent to treatment with fluorescein isothiocyanate (FITC)-conjugated goat anti-mouse IgG (1:100; sc-2010; Santa Cruz Biotechnology, Inc.) and PerCP-Cy5.5-conjugated goat anti-rabbit IgG (1:100; sc-45101; Santa Cruz Biotechnology, Inc.), fluorescence was observed under a fluorescent microscope (BX51; Olympus Corporation, Tokyo, Japan).

Flow cytometry analysis. Blood samples $(10 \mathrm{ml})$ were drawn using a needle and syringe from the peripheral vein of all patients, after which PBMs were isolated from the heparinized (Well-Biology) blood samples by Ficoll-Hypaque density gradient centrifugation at $150 \mathrm{x} \mathrm{g}$ and $4^{\circ} \mathrm{C}$ for $20 \mathrm{~min}$. Cells were then incubated at $4^{\circ} \mathrm{C}$ for $45 \mathrm{~min}$ in the dark with monoclonal PE-conjugated mouse anti-CD14 (1:10; sc-52457; Santa Cruz Biotechnology, Inc.) monoclonal FITC-conjugated mouse anti-HLA-DR (1:10; sc-33718; Santa Cruz Biotechnology, Inc.) and monoclonal PerCP-conjugated mouse anti-CD80 antibodies (1:10, sc-73382; Santa Cruz Biotechnology, Inc.). Samples were assayed using a FACSCalibur system (Beckman Coulter, Inc., Brea, CA, USA) and analysis was performed using CellQuest software, version 3.0 (BD Biosciences, Franklin Lakes, NJ, USA).

Phagocytic activity assay. According to the method described by Gille et al (15), monocytes were isolated from PBMs using CD14 MicroBeads ${ }^{\mathrm{TM}}$ (Miltenyi Biotec $\mathrm{GmbH}$, Bergisch Gladbach, Germany) and were passed through a MACS column (Miltenyi Biotec $\mathrm{GmbH}$ ) to positively select for $\mathrm{CD} 14^{+}$cells by immunomagnetic selection, according to the manufacturer's instructions. This procedure yielded a minimum of a $90 \%$ pure population of monocytes, as assessed by fluorescence-activated cell sorter analysis. The adherent monocytes were treated for $60 \mathrm{~min}$ in the dark with FITC-conjugated E. coli (a gift from Dr Jie Yin, Chinese Academy of Sciences), and were then washed with PBS and centrifuged at 3,000 x g and $4^{\circ} \mathrm{C}$ for $5 \mathrm{~min}$ to remove free bacteria. Samples were assayed using the FACSCalibur system. Phagocytic activity was expressed as a percentage of the FITC-conjugated cells.

Statistics. Data are presented as the mean \pm standard error. Significant differences between the means were evaluated by Student's t-test or analysis of variance. Spearman's rank correlation coefficient was used to examine the correlation. $\mathrm{P}<0.05$ was considered to indicate a statistically significant difference.

\section{Results}

Increased expression of VAVI and PAK1. The expression levels of VAV1 and PAK1 in the liver of patients with AIH and NAFLD controls were measured using western blotting. As presented in Table I, the expression levels of VAV1 and PAK1 were significantly increased in patients with AIH, as compared with the NAFLD group $(\mathrm{P}<0.05)$. In addition, a correlation between increased expression of VAV1 and PAK1 and an advanced disease stage was observed (data not shown).

The expression levels of VAV1 and PAK1 in KCs of AIH patients in comparison with NAFLD controls were additionally analyzed by immunofluorescence double staining (Figs. 1 and 2). Expression levels of VAV1 and PAK1 in KCs 
Table I. Western blotting of VAV1 and PAK1 expression in liver tissues of AIH and controls.

\begin{tabular}{lccc}
\hline Group & $\mathrm{N}$ & VAV1/GAPDH & PAK1/GAPDH \\
\hline NAFLD & 7 & $1.00 \pm 0.07$ & $1.00 \pm 0.08$ \\
AIH normal liver function & 7 & $1.79 \pm 0.78^{\mathrm{a}}$ & $1.64 \pm 0.52^{\mathrm{a}}$ \\
AIH abnormal liver function & 7 & $2.37 \pm 0.81^{\mathrm{a}}$ & $2.41 \pm 0.53^{\mathrm{a}}$ \\
AIH cirrhosis & 7 & $4.64 \pm 1.37^{\mathrm{a}}$ & $4.71 \pm 1.26^{\mathrm{a}}$ \\
\hline
\end{tabular}

${ }^{a} \mathrm{P}<0.05$ vs. NAFLD. PAK1, p21-activated kinase 1; AIH, autoimmune hepatitis; GAPDH, glyceraldehyde 3-phosphate dehydrogenase; NAFLD, non-alcoholic fatty liver disease.

A

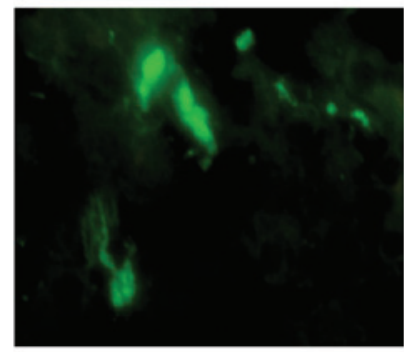

B

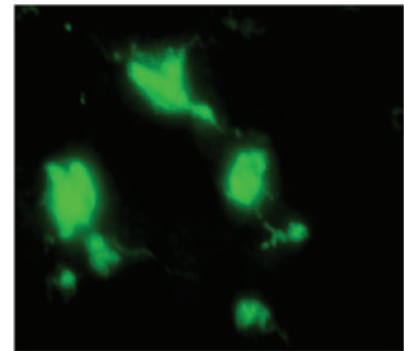

$\mathbf{C}$

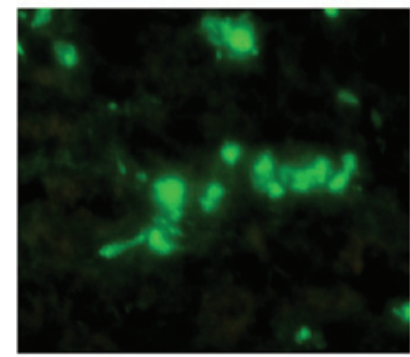

D

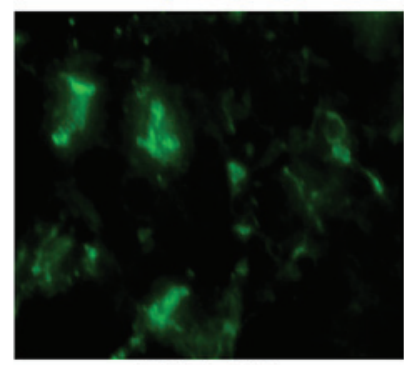

CD68
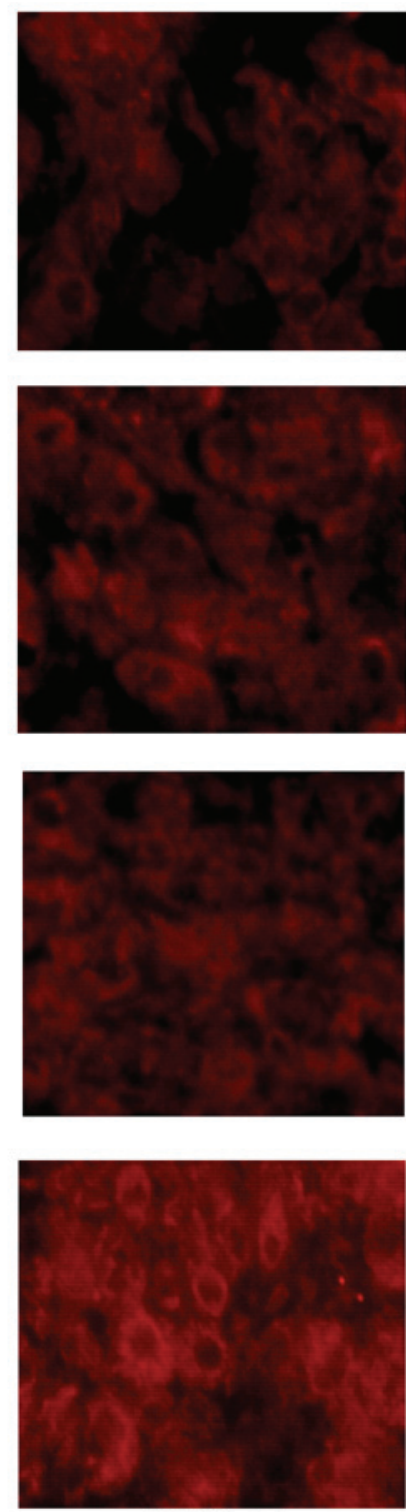

VAV1
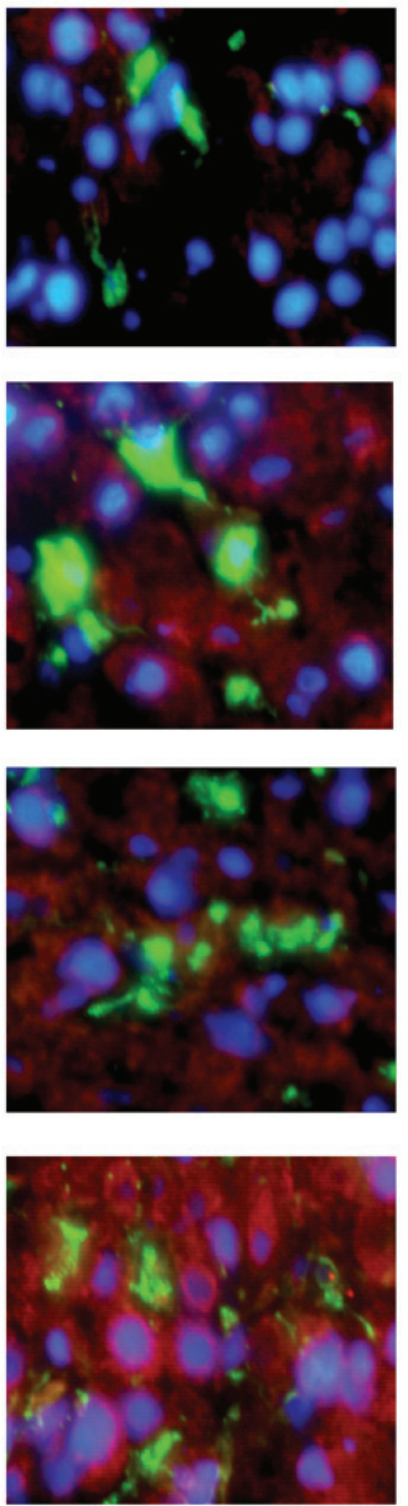

CD68+VAV1

Figure 1. Double immunostaining analysis of VAV1 expression in Kupffer cells (n=7). (A) NAFLD group. (B) AIH normal liver function group. (C) AIH abnormal liver function group. (D) AIH cirrhosis group. NAFLD, non-alcoholic fatty liver disease; AIH, autoimmune hepatitis.

were markedly increased in patients with AIH, as compared with the NAFLD group. In addition, a correlation between increased levels of VAV1 and PAK1 expression and an advanced disease stage was observed (data not shown).
Reduced expression of HLA-DR and CD80. As presented in Fig. 3A-E, the expression level of HLA-DR in CD14 ${ }^{+}$cells was $99.06 \pm 0.61 \%$ in the NAFLD group, $82.37 \pm 4.62 \%$ in the AIH normal liver function group, $73.54 \pm 8.53 \%$ in the AIH 
A

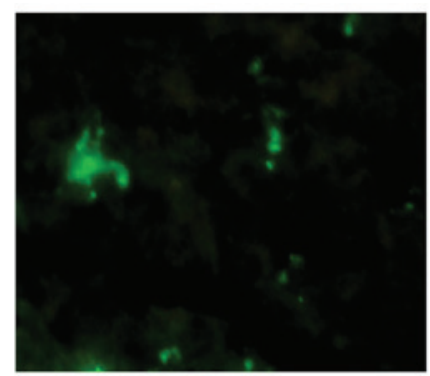

B

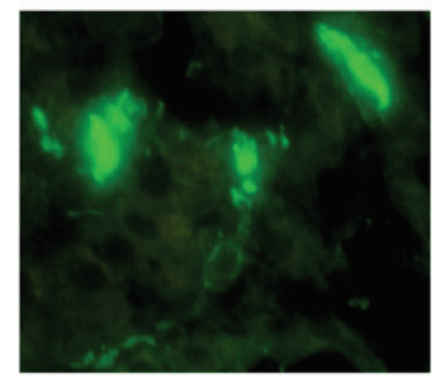

C

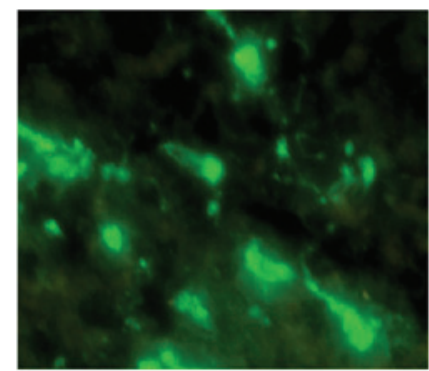

D

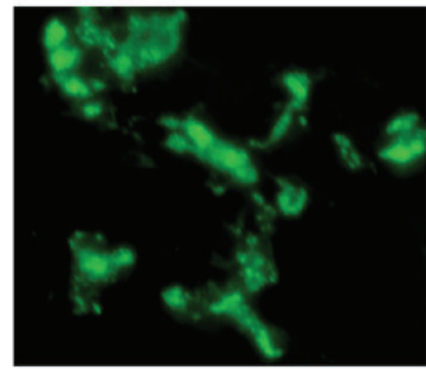

CD68
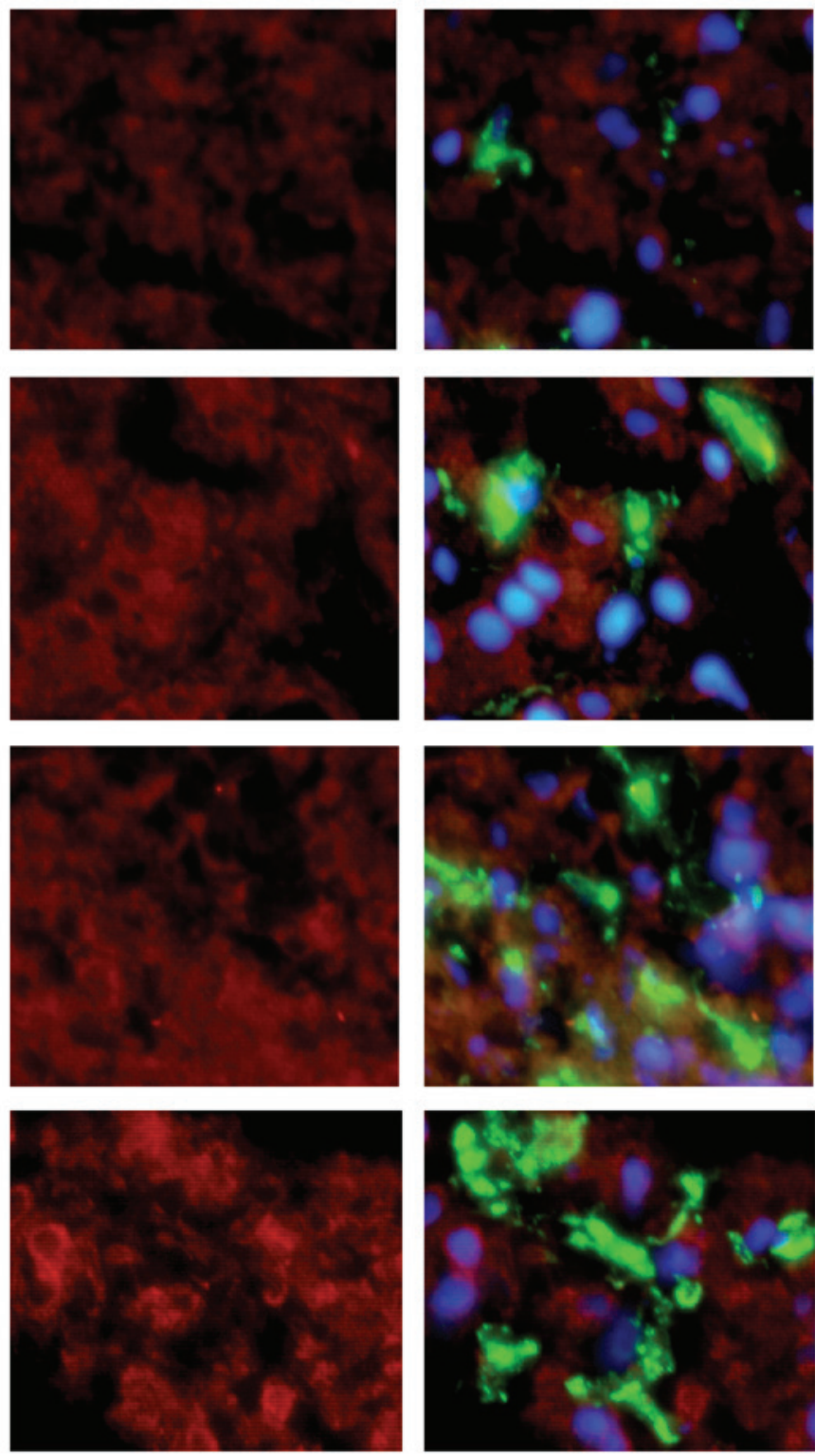

PAK1

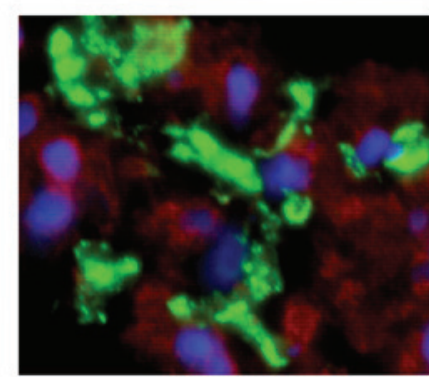

CD68+PAK1

Figure 2. Double immunostaining analysis of PAK1 expression in Kupffer cells (n=7). (A) NAFLD group. (B) AIH normal liver function group. (C) AIH abnormal liver function group. (D) AIH cirrhosis group. PAK1, p21-activated kinase 1; NAFLD, non-alcoholic fatty liver disease; AIH, autoimmune hepatitis.

abnormal liver function group and $60.63 \pm 11.47 \%$ in the $\mathrm{AIH}$ cirrhosis group. Cell surface expression of HLA-DR was significantly reduced on PBMs from patients with $\mathrm{AIH}$, as compared with those from NAFLD control subjects $(\mathrm{P}<0.05)$. In addition, a correlation between reduced expression of HLA-DR and an advanced disease stage was observed (data not shown).

As presented in Fig. 3F-J, the expression level of CD80 on $\mathrm{CD}_{14}{ }^{+}$cells was $81.46 \pm 5.08 \%$ in the NAFLD group, $69.15 \pm 11.37 \%$ in the AIH normal liver function group, $54.72 \pm 9.72 \%$ in the AIH abnormal liver function group and $43.24 \pm 15.52 \%$ in the AIH cirrhosis group. Cell surface expression of CD80 was significantly reduced on PBMs from patients with AIH, as compared with the NAFLD control subjects $(\mathrm{P}<0.05$; Fig. 3J). In addition, a correlation between reduced expression of CD80 and an advanced disease stage was observed (Fig. 3J).
Reduced phagocytic activity of PBMs. As presented in Fig. 4, the percentage of FITC-conjugated PBMs was $75.73 \pm 6.32 \%$ in the NAFLD group, $63.28 \pm 8.24 \%$ in the AIH normal liver function group, $52.69 \pm 10.36 \%$ in the AIH abnormal liver function group and $36.21 \pm 14.29 \%$ in the AIH cirrhosis group. Phagocytic activity was observed to be significantly reduced in monocytes from patients with $\mathrm{AIH}$, as compared with the NAFLD control subjects $(\mathrm{P}<0.05$; Fig. 4$)$. In addition, a correlation between reduction in phagocytic activity and an advanced disease stage was observed (Fig. 4).

\section{Discussion}

AIH is an inflammatory liver disease that is characterized by the presence of auto-antibodies, hypergammaglobulinemia, a histological evidence of interface hepatitis and an optimal response to steroids in the majority of patients (16). A 

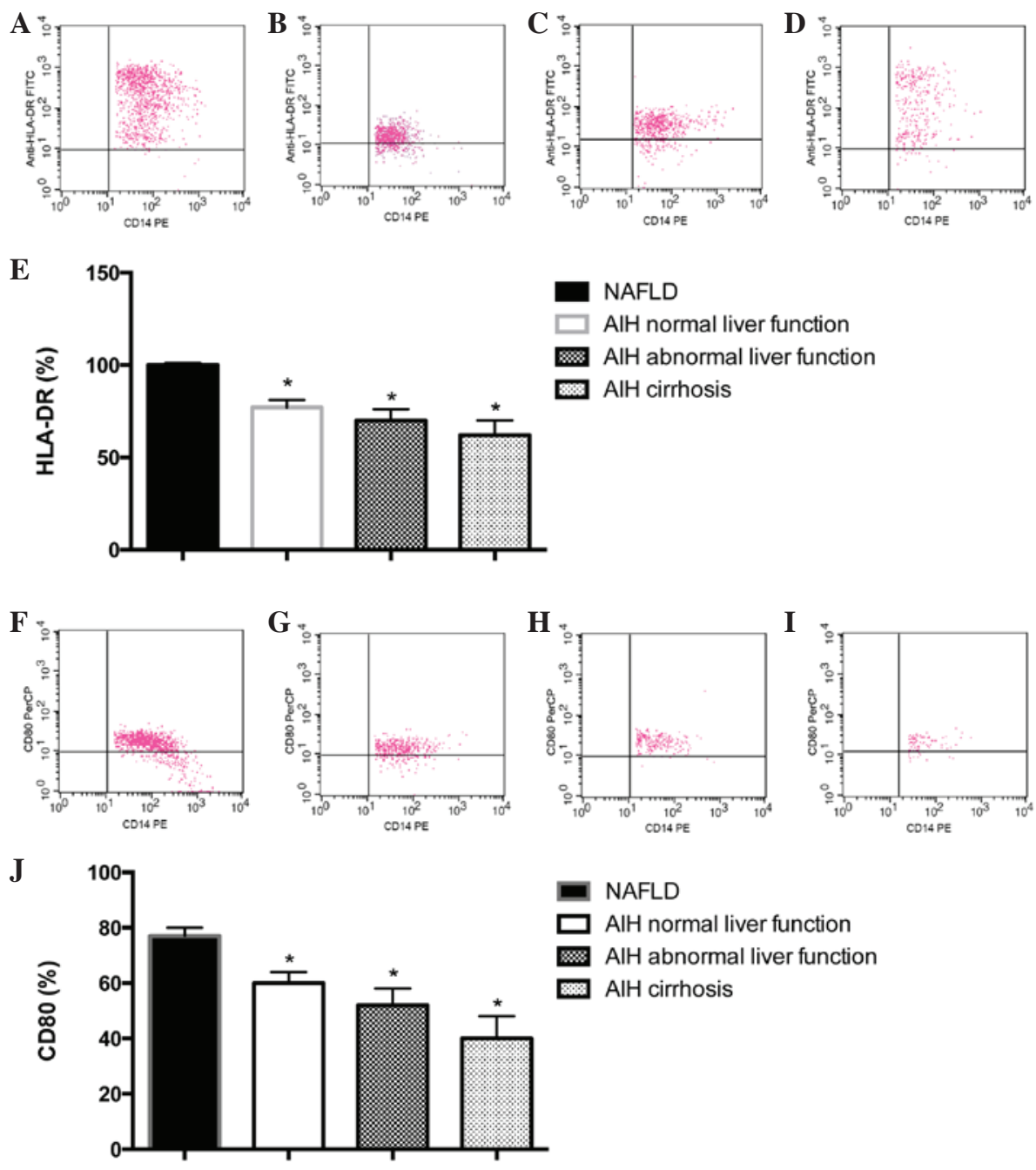

Figure 3. Flow cytometry analysis of HLA-DR and CD80 expression in peripheral blood monocytes of AIH and controls (n=7). HLA-DR expression in the (A) NAFLD group, (B) AIH normal liver function group, (C) AIH abnormal liver function group and (D) AIH cirrhosis group. (E) Quantification of the expression of HLA-DR in (A-D). CD80 expression in the (F) NAFLD group, (G) AIH normal liver function group, (H) AIH abnormal liver function group and (I) AIH cirrhosis group. (J) Quantification of the expression of CD80 in (F-I) * $\mathrm{P}<0.05$ vs. NAFLD. AIH, autoimmune hepatitis; NAFLD, non-alcoholic fatty liver disease.
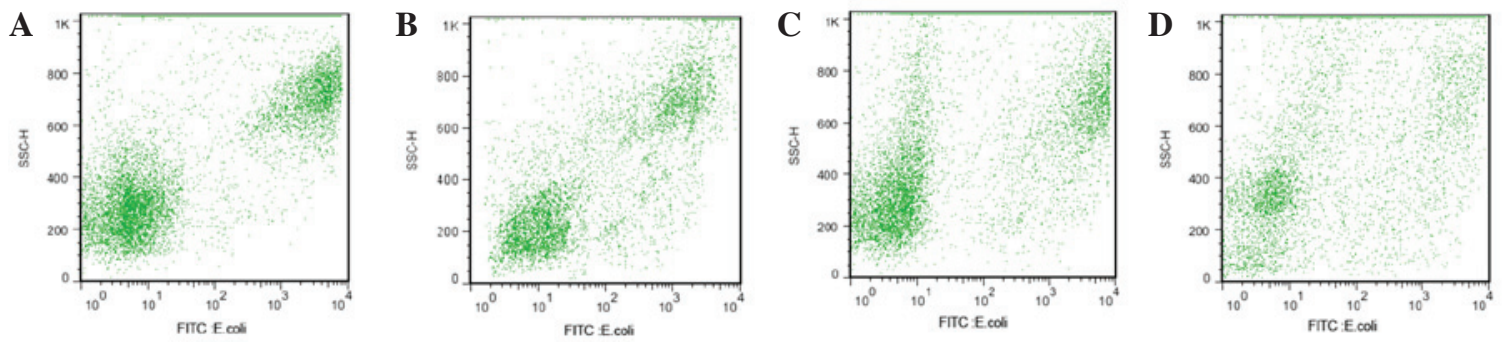

$\mathbf{E}$

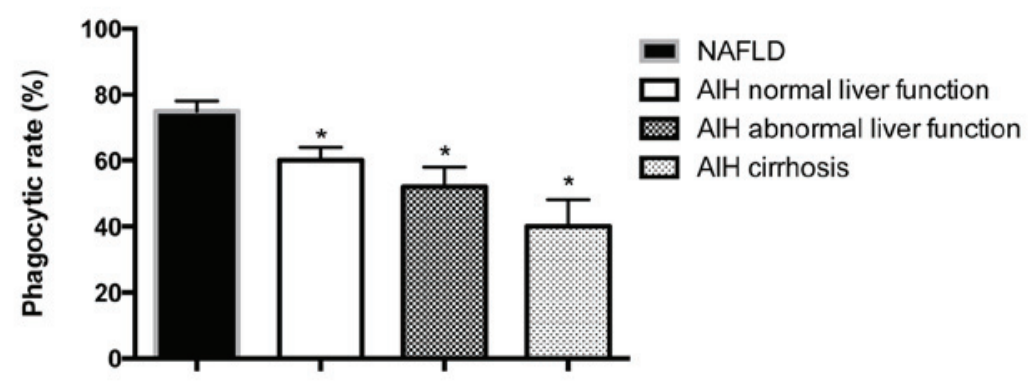

Figure 4. Phagocytic activity of peripheral blood monocytes was determined by uptake of FITC-conjugated E. coli (n=7). (A) NAFLD group. (B) AIH normal liver function group. (C) AIH abnormal liver function group. (D) AIH cirrhosis group. (E) Quantification of the expression of HLA-DR in (A-D). " $\mathrm{P}<0.05$ vs. NAFLD. FITC, fluorescein isothiocyanate; NAFLD, non-alcoholic fatty liver disease; AIH, autoimmune hepatitis; SSC, side scatter. 
previous study on the pathogenesis of AIH focused on the adaptive immune system, since lymphocytic abnormalities have previously been hypothesized to be the primary cause of autoimmunity (1). In the past decade, however, this focus has shifted with advances in the field of innate immunity. Monocytes/macrophages are a key component of the innate immune system with numerous immunological functions, including phagocytosis, antigen presentation and cytokine production. Phagocytosis is the process of the clearance of dead or dying cells, cellular debris, microbes and other foreign materials. The phagocytic capacity of monocytes/macrophages is essential for the host's defense against pathogens and homeostatic clearance of dead or dying cells. Monocytes/macrophages serve a pivotal role in the initiation of immunologically-mediated liver injury $(2,17)$. Phagocytic activity of peritoneal macrophages has been observed to be significantly impaired in mice with concanavalin A-induced hepatitis (18). Aberrant activation of macrophages may trigger inflammation that contributes to the initiation and progression of liver diseases. However, the function of monocytes/macrophages in patients with AIH remains to be fully elucidated.

KCs represent approximately $10 \%$ of the resting total liver cell population, however constitute the largest component (80 90\%) of all tissue macrophages in the body. Central to innate immunity, KCs are responsible for clearance of exogenous particulate and immunoreactive material, and antigen presentation, which aid in the maintenance of immune homeostasis (19). In the current study, KCs from patients with AIH were investigated. Obtaining liver biopsies from healthy individuals is unlikely to be approved by an institutional review board, thus in the current study, control KCs were obtained from patients with NAFLD. Due to the fact that the number of KCs present in a liver biopsy is too few to allow for conducting functional assays, and that monocytes serve as direct precursors to tissue macrophages, PBMCs were isolated to evaluate phagocytosis and antigen presenting functions. The phagocytic activity of PBMCs in patients with AIH was investigated with the FITC-conjugated $E$. coli phagocytosis assay. It was identified that phagocytosis was significantly impaired in PBMCs from patients with AIH compared with the NAFLD controls. This clearance defect may result in the accumulation of apoptotic cells, which can serve as antigens. Booth et al (8) demonstrated that the antigen-presentation capablities are closely associated with HLA-DR and CD80 expression levels. HLA-DR can provide an initial signal leading to the development of an effective immune response via formation of the specific peptide-major histocompatibility complex (MHC) antigen complex. CD80 is a co-stimulatory molecule present on activated monocytes and $\mathrm{B}$ cells and provides a co-stimulatory signal necessary for the activation and survival of $\mathrm{T}$ lymphocytes. In the current study, the expression levels of HLA-DR and CD80 on PBMCs were reduced in patients with AIH compared with the NAFLD individuals, suggesting an ineffective antigen-presenting function may contribute to an impaired antigen-specific immune response in these patients. These results support the theory that monocyte function may be defective, and thus may therefore be indicative of an impaired immune response status in patients with AIH.
Previous studies have demonstrated that Rho GTPases are the essential regulators of cell behavior, linking extracellular stimuli to intracellular signal transduction events $(20,21)$. The activity is controlled by guanine nucleotide exchange factors (GEF) that regulate the exchange from guanosine diphosphate (inactive Rho GTPase) to guanosine triphosphate (active Rho GTPase). Rho GTPases, including Cdc42, Rac, and Rho, then interact with downstream effectors to regulate cytoplasmic signaling pathways that control the vital cellular processes, including cytoskeletal dynamics, cell cycle progression, gene transcription and cell transformation (22). Antigen presentation is associated with phagocytosis, which occurs via remodeling of the actin cytoskeleton and shares numerous core cytoskeletal components involved in adhesion and migration (23). VAV1 is a GEF for Rho GTPases and is able to regulate the activation of Rac, Rho and Cdc42 (24). It has been reported that VAV1 may serve a crucial role in actin rearrangement at the phagocytic cup and MHC II expression in dendritic cells and macrophages $(25,26)$. PAK1, a serine/threonine kinase, has been identified as a major downstream effector of the Rho GTPases Rac1 and Cdc42 (27). PAK1 serves an essential role in regulating cellular processes such as cytoskeletal remodeling, cell motility, cell proliferation and cell survival (28). The expression levels of VAV1 and PAK1 on KCs were observed to be significantly increased in patients with AIH. The PBMC functions of phagocytosis and antigen-presentation were reduced, and correlated with the disease progression. This may have been due to aberrant activation of Rho GTPase signaling associated with the breakdown of actin patches and the delayed closure of the phagocytic cup (29), which induces dysfunction of monocytes/macrophages.

Taken together, these data suggest a potential mechanism for Rho GTPases in the regulation of the function of monocytes/macrophages. Liver damage may result from the inability of KCs to eliminate immunoreactive materials, which are then able to initiate the immune process. These observations aid in the elucidation of the pathogenesis of $\mathrm{AIH}$, and may provide a novel therapeutic target. Due to the small number of patients enrolled in the current study and the limitations of the biopsy tissues, further investigation into the precise signaling pathway of Rho GTPases, and how these are involved in the regulation of $\mathrm{KC}$ function in an $\mathrm{AIH}$ model are required.

\section{References}

1. Ma Y, Bogdanos DP, Hussain MJ, Underhill J, Bansal S, Longhi MS, Cheeseman P, Mieli-Vergani G and Vergani D: Polyclonal T-cell responses to cytochrome P450IID6 are associated with disease activity in autoimmune hepatitis type 2 . Gastroenterology 130: 868-882, 2006.

2. Seki E and Schnabl B: Role of innate immunity and the microbiota in liver fibrosis: Crosstalk between the liver and gut. J Physiol 590: 447-458, 2012.

3. Lleo A, Bowlus CL, Yang GX, Invernizzi P, Podda M, Van de Water J, Ansari AA, Coppel RL, Worman HJ, Gores GJ and Gershwin ME: Biliary apotopes and anti-mitochondrial antibodies activate innate immune responses in primary biliary cirrhosis. Hepatology 52: 987-998, 2010.

4. Bouwens L, Baekeland M, De Zanger R and Wisse E: Quantitation, tissue distribution and proliferation kinetics of Kupffer cells in normal rat liver. Hepatology 6: 718-722, 1986.

5. Baffy G: Kupffer cells in non-alcoholic fatty liver disease: The emerging view. J Hepatol 51:212-223, 2009. 
6. Burdo TH, Lackner A and Williams KC: Monocyte/macrophages and their role in HIV neuropathogenesis. Immunol Rev 254: 102-113, 2013

7. Pul R, Morbiducci F, Škuljec J, Skripuletz T, Singh V, Diederichs U, Garde N, Voss EV, Trebst C and Stangel M: Glatiramer acetate increases phagocytic activity of human monocytes in vitro and in multiple sclerosis patients. PLoS One 7: e51867, 2012.

8. Booth S, Florida-James GD, McFarlin BK, Spielmann G, O'Connor DP and Simpson RJ: The impact of acute strenuous exercise on TLR2, TLR4 and HLA.DR expression on human blood monocytes induced by autologous serum. Eur J App Physiol 110: 1259-1268, 2010.

9. Wang D, Yuan F, Wang L and Wei W: Paeoniflorin inhibits function and down-regulates HLA-DR and CD80 expression of human peripheral blood monocytes stimulated by rhIL-1 $\beta$. Int Immunopharmacol 14: 172-178, 2012.

10. Jennings RT and Knaus UG: Rho family and Rap GTPase activation assays. Methods Mol Biol 1124: 79-88, 2014.

11. Katzav S: Vav1:A hematopoietic signal transduction molecule involved in human malignancies. Int $\mathrm{J}$ Biochem Cell Biol 41:1245-1248, 2009.

12. Jhaveri KA, Debnath P, Chernoff J, Sanders J and Schwartz MA: The role of $\mathrm{p} 21$-activated kinase in the initiation of atherosclerosis. BMC Cardiovasc Disord 12: 55, 2012

13. Hennes EM, Zeniya M, Czaja AJ, Parés A, Dalekos GN, Krawitt EL, Bittencourt PL, Porta G, Boberg KM Hofer H, et al: Simplified criteria for the diagnosis of autoimmune hepatitis. Hepatology 48: 169-176, 2008.

14. Chalasani N, Younossi Z, Lavine JE, Diehl AM, Brunt EM, Cusi K, Charlton M and Sanyal AJ; American Gastroenterological Association; American Association for the Study of Liver Diseases; American College of Gastroenterologyh: The diagnosis and management of non-alcoholic fatty liver disease: Practice guideline by the American gastroenterological association, American association for the study of liver diseases and American college of gastroenterology. Gastroenterology 142: 1592-1609, 2012 .

15. Gille C, Leiber A, Mundle I, Spring B, Abele H, Spellerberg B, Hartmann H, Poets ChF and Orlikowsky TW: Phagocytosis and postphagocytic reaction of cord blood and adult blood monocyte after infection with green fluorescent protein-labeled Escherichia coli and group B Streptococci. Cytometry B Clin Cytom 76: 271-284, 2009.

16. Liberal R, Longhi MS, Mieli-Vergani G and Vergani D Pathogenesis of autoimmune hepatitis. Best Pract Res Clin Gastroenterol 25: 653-664, 2011.
17. Heymann F, Hammerich L, Storch D, Bartneck M, Huss S, Rüsseler V, Gassler N, Lira SA, Luedde T, Trautwein C and Tacke F: Hepatic macrophage migration and differentiation critical for liver fibrosis is mediated by the chemokine receptor $\mathrm{C}-\mathrm{C}$ motif chemokine receptor 8 in mice. Hepatology 55: 898-909, 2012.

18. Yu Z, Otsuka H, Yamaguchi K, Kuroishi T, Sasano T, Sugawara S, Nakamura M and Endo Y: Roles of platelets and macrophages in the protective effects of lipopolysaccharide against concanavalin A-induced murine hepatitis. Biochim Biophys Acta 1812: 1069-1079, 2011.

19. Naito M, Hasegawa G and Takahashi K: Development, differentiation and maturation of Kupffer cells. Microsc Res Tech 39: 350-364, 1997.

20. Etienne-Manneville S and Hall A: Rho GTPases in cell biology. Nature 420: 629-635, 2002

21. Swetman CA, Leverrier Y, Garg R, Gan CH, Ridley AJ, KatzDR and Chain BM: Extension, retraction and contraction in the formation of a dendritic cell dendrite: Distinct roles for Rho GTPases. Eur J Immunol 32: 2074-2083, 2002.

22. Ridley AJ: Rho proteins, PI 3-kinases and monocyte/macrophage motility. FEBS Lett 498: 168-171, 2001.

23. Tse SM, Furuya W, Gold E, Schreiber AD, Sandvig K, Inman RD and Grinstein S: Differential role of actin, clathrin and dynamin in Fc gamma receptor-mediated endocytosis and phagocytosis. J Biol Chem 278: 3331-3338, 2003

24. Chrencik JE, Brooun A, Zhang H, Mathews II, Hura GL, Foster SA, Perry JJ, Streiff M, Ramage P, Widmer H, et al: Structural basis of guanine nucleotide exchange mediated by the T-cell essential Vav1. J Mol Biol 380: 828-843, 2008.

25. Wells CM, Bhavsar PJ, Evans IR, Vigorito E, Turner M, Tybulewicz V and Ridley AJ: Vav1 and Vav2 play different roles in macrophage migration and cytoskeletal organization. Exp Cell Res 310: 303-310, 2005.

26. de la Fuente H, Mittelbrunn M, Sánchez-Martín L, Vicente-Manzanares M, Lamana A, Pardi R, Cabañas C and Sánchez-Madrid F: Synaptic clusters of MHC class II molecules induced on DCs by adhesion molecule-mediated initial T-cell scanning. Mol Biol Cell 16: 3314-3322, 2005.

27. Arias-Romero LE and Chernoff J: A tale of two Paks. Biol Cell 100: 97-108, 2008

28. Delorme V, Machacek M, DerMardirossian C, Anderson KL, Wittmann T, Hanein D, Waterman-Storer C, Danuser G and Bokoch GM: Cofilin activity downstream of Pak1 regulates cell protrusion efficiency by organizing lamellipodium and lamella actin networks. Dev Cell 13: 646-662, 2007.

29. Hsu HY and Twu YC: Tumor necrosis factor-alpha-mediated protein kinases in regulation of scavenger receptor and foam cell. J Biol Chem 275: 41035-41048, 2000. 